\title{
Synchrony, metastability, dynamic integration, and competition in the spontaneous functional connectivity of the human brain
}

\author{
Vincent Wens ${ }^{\mathrm{a}, \mathrm{b}, *}$, Mathieu Bourguignon ${ }^{\mathrm{a}, \mathrm{c}, \mathrm{d}}$, Marc Vander Ghinst ${ }^{\mathrm{a}}$, Alison Mary ${ }^{\mathrm{e}}$, Brice Marty ${ }^{\mathrm{a}}$, \\ Nicolas Coquelet $^{a}$, Gilles Naeije ${ }^{\mathrm{a}}$, Philippe Peigneux ${ }^{\mathrm{f}}$, Serge Goldman ${ }^{\mathrm{a}, \mathrm{b}}$, Xavier De Tiège ${ }^{\mathrm{a}, \mathrm{b}}$ \\ ${ }^{\text {a }}$ LCFC - Laboratoire de Cartographie fonctionnelle du Cerveau, UNI - ULB Neuroscience Institute, Université libre de Bruxelles (ULB), Brussels, Belgium \\ ${ }^{\mathrm{b}}$ Magnetoencephalography Unit, Department of Functional Neuroimaging, Service of Nuclear Medicine, CUB - Hôpital Erasme, Brussels, Belgium \\ ${ }^{\mathrm{c}}$ Laboratoire Cognition Langage et Développement, UNI - ULB Neuroscience Institute, Université libre de Bruxelles (ULB), Brussels, Belgium \\ ${ }^{\mathrm{d}}$ BCBL - Basque Center on Cognition, Brain and Language, 20009 San Sebastian, Spain \\ ${ }^{\text {e } U N I C A E N, ~ P S L ~ R e s e a r c h ~ U n i v e r s i t y, ~ E P H E, ~ I N S E R M, ~ U 1077, ~ C H U ~ d e ~ C a e n, ~ N e u r o p s y c h o l o g i e ~ e t ~ I m a g e r i e ~ d e ~ l a ~ M e ́ m o i r e ~ H u m a i n e, ~ C a e n, ~ F r a n c e ~}$ \\ ${ }^{\mathrm{f}}$ UR2NF - Neuropsychology and Functional Neuroimaging Research Unit at CRCN - Centre de Recherches Cognition et Neurosciences, UNI - ULB Neuroscience Institute, \\ Université libre de Bruxelles (ULB), Brussels, Belgium
}

\section{A R T I C L E I N F O}

\section{Keywords:}

Connectivity state transition

Dynamic functional connectivity

Independent component analysis

Magnetoencephalography

Network mixture model

Resting state

\begin{abstract}
A B S T R A C T
The human brain is functionally organized into large-scale neural networks that are dynamically interconnected. Multiple short-lived states of resting-state functional connectivity (rsFC) identified transiently synchronized networks and cross-network integration. However, little is known about the way brain couplings covary as rsFC states wax and wane. In this magnetoencephalography study, we explore the synchronization structure among the spontaneous interactions of well-known resting-state networks (RSNs). To do so, we extracted modes of dynamic coupling that reflect rsFC synchrony and analyzed their spatio-temporal features. These modes identified transient, sporadic rsFC changes characterized by the widespread integration of RSNs across the brain, most prominently in the $\beta$ band. This is in line with the metastable rsFC state model of resting-state dynamics, wherein our modes fit as state transition processes. Furthermore, the default-mode network (DMN) stood out as being structured into competitive cross-network couplings with widespread DMN-RSN interactions, especially among the $\beta$-band modes. These results substantiate the theory that the DMN is a core network enabling dynamic global brain integration in the $\beta$ band.
\end{abstract}

\section{Introduction}

The large-scale organization of the human brain is based on the existence of functional networks, which reflect the preferential integration of distant neural assemblies needed to support various functions, from sensory perception and motor behaviors to complex cognitive processes. At the macroscopic level, spatial patterns of interaction between segregated brain areas can be disclosed during the performance of dedicated tasks, but also in the absence of any explicit task (i.e., the so-called resting state). These background couplings are thought to represent a fingerprint of this organization into functional networks and to reflect the intrinsic functional architecture of the human brain (for a review, see, e.g., Deco and Corbetta, 2011).

One method to map functional brain networks from experimental measurements is functional connectivity (FC) analysis, where the coupling among network nodes is estimated via measures of statistical similarity between their activities (Bastos and Schoffelen, 2015; Friston, 2011; O'Neill et al., 2015a). This approach generally assumes that networks are temporally stable. Multiple static interaction patterns - conventionally referred to as resting-state networks (RSNs) - have been identified in this way, some overlapping primary systems such as the sensorimotor, the auditory, and the visual networks and others involving higher-level systems such as the attentional, the executive-control, and the default-mode networks. These observations have been consistently reproduced across neuroimaging studies and modalities, from functional magnetic resonance imaging (fMRI) (Beckmann et al., 2005; Biswal et al., 1995; Damoiseaux et al., 2006; Fox et al., 2005; Smith et al., 2009) to magnetoencephalography (MEG) (Brookes

\footnotetext{
* Corresponding author. Magnetoencephalography Unit, Department of Functional Neuroimaging, Service of Nuclear Medicine, CUB - Hôpital Erasme, 808 route de Lennik, 1070 Brussels, Belgium.

E-mail address: vwens@ulb.ac.be (V. Wens).
} 
et al., 2011, 2012a, b; Hall et al., 2013; Hipp et al., 2012; Liu et al., 2010; Luckhoo et al., 2012; Wens et al., 2014a, b, 2015) and electroencephalography (EEG) (Knyazev et al., 2016, 2017; Liu et al., 2017; Siems et al., 2016; Sockeel et al., 2016).

However, this static picture of RSNs-i.e., fixed networks without time-dependent coupling modulations within and across them-hardly mirrors the functional landscape of the human brain. Rather, it has been hypothesized that RSNs represent an average of temporally varying interaction patterns, which would transiently fragment into subnetworks to bind different systems together, hence bringing about a richer repertoire of functional integration (Deco et al., 2011). This hypothesis was supported by dynamic FC studies, which disclosed a large body of time-dependent interaction patterns fluctuating over relatively short timescales (from seconds to tens of seconds), including transient cross-network couplings (Allen et al., 2014; Brookes et al., 2014; Chang and Glover, 2010; de Pasquale et al., 2010, 2012; Handwerker et al., 2012; Hutchison et al., 2012; Kiviniemi et al., 2011; O'Neill et al., 2015b, 2017b; Zalesky et al., 2014). Still, many aspects of dynamic FC remain to be investigated (for reviews, see Hutchison et al., 2013; O'Neill et al., 2017a).

In this work, we explore the existence and the properties of spontaneous temporal synchrony among dynamic brain couplings and consider the hypothesis that coupling synchronization is a correlate of the generation of sub-networks and cross-network integration. We started with resting-state FC measured via MEG power envelope correlation from key nodes of well-established RSNs, and submitted the time-dependent FC data to an independent component analysis (ICA) so as to disclose "modes of dynamic coupling" that reflect patterns of synchronous fluctuation among brain interactions. This approach has already been used to disclose task-related transiently synchronized networks (O'Neill et al., 2017b). An extra challenge here was to identify physiologically relevant modes of dynamic coupling in the absence of any controlled, goal-directed task. To do this and test our main hypothesis, we sought to relate each mode to a combination of template RSNs (henceforth referred to as "network mixture models"). This would establish an association between coupling synchrony and the phenomenon of cross-RSN integration. The framework of network mixture modeling also allows to investigate the key hypothesis that resting-state activity emerges from a spontaneous switching among diverse network configurations, which can be interpreted effectively as a "dynamical competition" among RSNs (Deco and Corbetta, 2011). We sought to identify competitive modes of dynamic coupling by comparing their mixture model to a similar model explicitly constrained to display no competition. Establishing their existence would provide empirical support to the theory of Deco and Corbetta (2011).

\section{Material and methods}

\subsection{Data acquisition}

The dataset used in this work consists of MEG resting-state recordings of 100 healthy adult volunteers ( 48 females and 52 males, mean age: 26.5 years, age range: 18-41 years) gathered from nine experiments containing a rest session ( $5 \mathrm{~min}$, eyes open) intermingled with task-driven sessions. Results related to task-positive phenomena have been published previously (Bourguignon et al., 2011, 2013; Clumeck et al., 2014; Marty et al., 2015; Mary et al., 2015; Vander Ghinst et al., 2016). Participants were all right-handed as assessed by the Edinburgh Handedness Inventory (Oldfield, 1971), had no history of neurologic or psychiatric disease, and signed a written informed consent prior to data acquisition. All nine studies were approved by the CUB - Hôpital Erasme Ethics Committee.

Neuromagnetic activity at rest was acquired using a 306-channel whole-scalp-covering MEG system (Vectorview, Elekta Oy, Helsinki, Finland) placed in a lightweight magnetically shielded room (Maxshield $^{\mathrm{TM}}$, Elekta Oy, Helsinki, Finland). Signals were band-pass filtered at
$0.1-330 \mathrm{~Hz}$ and sampled at $1 \mathrm{kHz}$. Subjects were sitting comfortably in the MEG armchair with the head inside the MEG helmet, and were asked to relax and fixate the gaze at a point on the wall or on a screen. Their head position was tracked with four indicator coils. An electromagnetic digitalization system (Fastrack, Polhemus, Colchester, VT, USA) was used before MEG data acquisition to locate these coils relative to anatomical fiducials, as well as at least 150 head-surface points. A high-resolution 3D T1-weighted cerebral magnetic resonance image (MRI) of each subject was also acquired using a $1.5 \mathrm{~T}$ MRI scanner (Intera, Philips, The Netherlands) after the MEG recordings.

\subsection{Data preprocessing}

Resting-state MEG data were preprocessed offline for noise reduction and extraction of band-limited activity. The temporal extension of signal space separation was first applied using the Maxfilter software (MaxfilterTM, Elekta Oy, Helsinki, Finland; version 2.2 with default parameters) in order to suppress external magnetic interferences and correct for head movements (Taulu et al., 2005). Remaining cardiac, ocular, and system artifacts were then removed via an ICA (FastICA algorithm with dimension reduction to 30 and nonlinearity tanh, see Hyvärinen and Oja, 2000) applied to band-pass filtered $(0.5-45 \mathrm{~Hz})$ MEG signals. Components corresponding to these artifacts were visually selected (number of identified components per subject: $5.0 \pm 1.4$, mean \pm SD) and projected out of the full-rank data (Vigario et al., 2000). Of note, this ICA decomposition did not isolate high-frequency muscle artifacts, but their contribution below $45 \mathrm{~Hz}$ was subdominant. The cleaned MEG data were finally filtered in four frequency bands $\left(\theta: 4-8 \mathrm{~Hz}, \alpha: 8-12 \mathrm{~Hz}, \beta: 12-21 \mathrm{~Hz}, \beta^{\prime}\right.$ : $21-30 \mathrm{~Hz}$ ) and Hilbert transformed to obtain their analytic signals.

Individual MRIs were also preprocessed in order to build the MEG forward model needed for source reconstruction. First, the coordinate system associated with MEG was coregistered manually to that of MRI using the digitized fiducials for initial estimation and the head-surface points for manual refinements. The MRI was then segmented using the FreeSurfer image analysis suite (Fischl, 2012). Sources in three orthogonal directions were also placed at each node of a cortically-constrained grid (inter-sources distance: $5 \mathrm{~mm}$, total number of nodes: 13229). The grid was built on the Montreal Neurological Institute (MNI) template MRI and mapped onto each individual MRI via a non-linear spatial deformation algorithm implemented in the SPM8 toolbox (Friston et al., 2007). Finally, the MEG forward model was computed at these sources using the one-layer boundary element method of the MNE-C software suite (Gramfort et al., 2014).

\subsection{Sliding-window connectivity estimation}

We evaluated FC between the main nodes of RSNs and the rest of the cortex using sources envelope correlation, which was chosen here for its established ability to uncover all RSNs typically disclosed with fMRI (de Pasquale et al., 2010; Brookes et al., 2011; Hipp et al., 2012; Wens et al., 2014b). The FC analysis used here relied on minimum norm estimation (MNE) for source reconstruction and the geometric correction scheme for the suppression of spatial leakage effects, which yield spurious contributions to both static and dynamic FC (Wens, 2015). The pipeline has been described in Wens et al. (2015), to which we refer for further details. The main difference is that FC was estimated here within short time windows sliding across the recording (i.e., dynamic FC) rather than within a single window covering the entire recording (i.e., static FC). Specifically, the correlation between source envelopes (low-pass filtered at $2 \mathrm{~Hz}$ to improve the connectivity-to-noise ratio; see de Pasquale et al., 2010; Hipp et al., 2012) was estimated within 10s-long windows (de Pasquale et al., 2010) sliding with a step of 5 s, leading to 57 windows per subject. Note that leakage-corrected dynamic FC was computed using one-dimensional projected sources (as in O'Neill et al., 2015b, 2017b) rather than the Euclidean norm of three-dimensional sources (as in Betti et al., 2018). 
The seed sources used for the computation of seed-based dynamic FC were chosen as key nodes of six well-known RSNs: the default-mode (DMN), the sensorimotor (SMN), the auditory (AN), the visual (VN), and the left and right fronto-parietal ( $\mathrm{L} / \mathrm{RFPN}$ ) networks (see Table 1). The resulting RSN-specific sets of seed-based envelope correlation maps fluctuating from window to window represent the time development of couplings between each RSN node and the rest of the cortex.

\subsection{Identification of modes of dynamic coupling}

To detect modes of dynamic coupling that reflect synchronous fluctuations in the dynamic FC of each RSN, we assumed these modes to be mutually temporally independent (asynchronous) and thus estimated them using group ICA decomposition, as in O'Neill et al. (2017b). The method is illustrated in the top part of Fig. 1. Further background is provided in the Supplementary Methods S1 to S3.

To design a group-level analysis, we first standardized individual FC time series to zero mean (to ensure that the sought dynamical effects are not confounded with inter-individual variability in static FC) and unit variance (to ensure that each individual subject is given a similar weight) and concatenated them temporally across subjects. We also concatenated spatially the seed-based maps associated with the nodes of the RSN under consideration (see Table 1) in order to consider all its interactions at once. The resulting FC dataset was then decomposed into temporally independent components (ICs), each IC thus representing one mode of dynamic coupling. Parameters of the ICA were similar to those used in static FC analyses of MEG resting-state data (Brookes et al., 2011; Wens et al., 2014b). The dimensionality was first reduced to 50, which removed approximately $50 \%$ of the total variance in all cases. Of note, a similar proportion of variance was discarded as well in the previous MEG envelope ICA that successfully disclosed RSNs (see Supplementary Results S1). The ICA itself was performed using the FastICA algorithm with nonlinearity tanh and the number of ICs to compute was set to 20 (Hyvärinen and Oja, 2000).

We next proceeded with a detailed characterization of the modes of dynamic coupling. Each mode was associated with one IC time series and multiple IC spatial maps (one per seed belonging to the RSN under consideration). The maps were obtained by temporal correlation between FC and IC time series (see the raw IC maps in Fig. 1). We then applied various statistical analyses to

(i) identify for each mode the spatial location and the temporal characteristics of the couplings involved,

(ii) decompose its IC maps into combinations of template RSNs using a network mixture model, and

Table 1

List of seed locations used in dynamic FC mapping. Coordinates were taken from de Pasquale et al. (2012) and Hipp et al. (2012). PCC: posterior cingulate cortex; MPFC: mesio-prefrontal cortex; TPJ: temporo-parietal junction; SM: sensorimotor; A: auditory; V: visual; FEF: frontal eye field; IPS: intra-parietal sulcus; L: left; R: right.

\begin{tabular}{lll}
\hline seed RSN & key nodes & MNI coordinates (mm) \\
\hline DMN & PCC & $-3,-54,31$ \\
& MPFC & $-2,51,2$ \\
& LTPJ & $-43,-76,35$ \\
\multirow{2}{*}{ SMN } & RTPJ & $51,-64,32$ \\
& LSM & $-42,-26,54$ \\
AN & RSM & $38,-32,48$ \\
& LA & $-54,-22,10$ \\
VN & RA & $52,-24,12$ \\
& LV & $-20,-86,18$ \\
LFPN & RV & $16,-80,26$ \\
& LFEF & $-26,-12,53$ \\
RFPN & LIPS & $-25,-67,48$ \\
& RFEF & $30,-13,53$ \\
& RIPS & $23,-69,49$ \\
\hline
\end{tabular}

(iii) determine whether the mode is indicative of a "competitive" behavior between RSNs.

\subsection{Spatial mapping and temporal properties}

To locate the couplings involved in each mode, we masked all IC maps statistically using a parametric correlation test based on the null hypothesis that Fisher-transformed correlation values follow a normal distribution with mean zero and SD $1 / \sqrt{\nu-3}$. Here, the number of temporal degrees of freedom $\nu$ was estimated as the total number of time windows across subjects divided by two to take into account windows overlap. The significance level was set to $p<0.05$ with the family-wise error rate (FWER) controlled via Bonferroni correction for the $S$ seeds (see Table 1), the twenty ICs, and the effective number (here, $\rho=46$ ) of independent cortical sources estimated as the rank of the MEG forward model (see Wens et al., 2015). Both positive and negative correlations were considered (two-tailed test), leading to significance thresholds of \pm 0.081 (when $S=4$ ) and \pm 0.078 (when $S=2$ ) used to mask the IC maps (see the masked IC maps in Fig. 1). The volume fraction (across the $S$ maps) of these statistical masks was used to assess the cortical coverage of those couplings identified for each mode. Note that our simple approach might a priori overestimate the parameter $\nu$ (because of temporal autocorrelations in FC time series) and lead to lenient statistical masking. However, this was not the case, as estimates based on Fourier-phase surrogates, which preserve temporal autocorrelation, led to lower statistical thresholds (about \pm 0.06 ).

We also examined some temporal characteristics of the IC time series (see Fig. 1). To detect possible oscillatory dynamics, their power spectral density was estimated by applying the discrete Fourier transform to the single-subject parts of these time series and group averaging of the resulting magnitude-squared Fourier coefficients. We also considered higher-order statistics of IC time series, i.e., their skewness and kurtosis. Significance was assessed using a parametric test based on the null, approximately normal distribution obtained in the case of Gaussian signals. The null mean and SD were respectively zero and $\sqrt{6 / \nu}$ for the skewness, and 3 and $\sqrt{24 / \nu}$ for the kurtosis. The significance level was set to $p<0.05$ with Bonferroni correction for the twenty ICs. We tested for both positive and negative skewness (two-tailed test, threshold: \pm 0.14 ) and for the excess kurtosis (i.e., one-tailed test above 3, threshold: 3.26).

It is useful to explain how the skewness and the kurtosis inform the interpretation of the modes of dynamic coupling. The IC skewness elucidates the meaning of maps sign. Indeed, positivity and negativity have no absolute meaning because of the sign ambiguity inherent to ICA (Hyvärinen and Oja, 2000) but they do have a relative meaning among the four maps and the skewness. Within a given mode, two connections (i.e., two seed-target pairs) with the same sign in the corresponding IC maps represent two couplings that fluctuate in synchrony. If they have opposite sign, the two couplings are anticorrelated, which hints at a "dynamical competition" in this mode. A positive (negative) skewness indicates a tendency of the mode to increase connections with positive (negative) map values and concomitantly decrease those with opposite sign, whereas a vanishing skewness indicates the absence of such a preferential direction in FC changes.

The IC excess kurtosis estimates to what extent these FC modulations arise in sharp peaks but does not inform on the temporal unfolding of these peaks per se. To quantify their transient character, we used the mean lifetime of large-deviation events in IC time series. The identification of those large deviations driving the IC kurtosis was based on a threshold defined heuristically as follows. We increased candidate thresholds from 1 by step of 0.5 (recall here that IC time series are standardized to zero mean and unit SD), computed for each mode, RSN, and frequency band the probability of supra-threshold events within IC time series, and retained the smallest threshold for which there was a significantly positive $(p<0.05)$ Pearson correlation (across the 


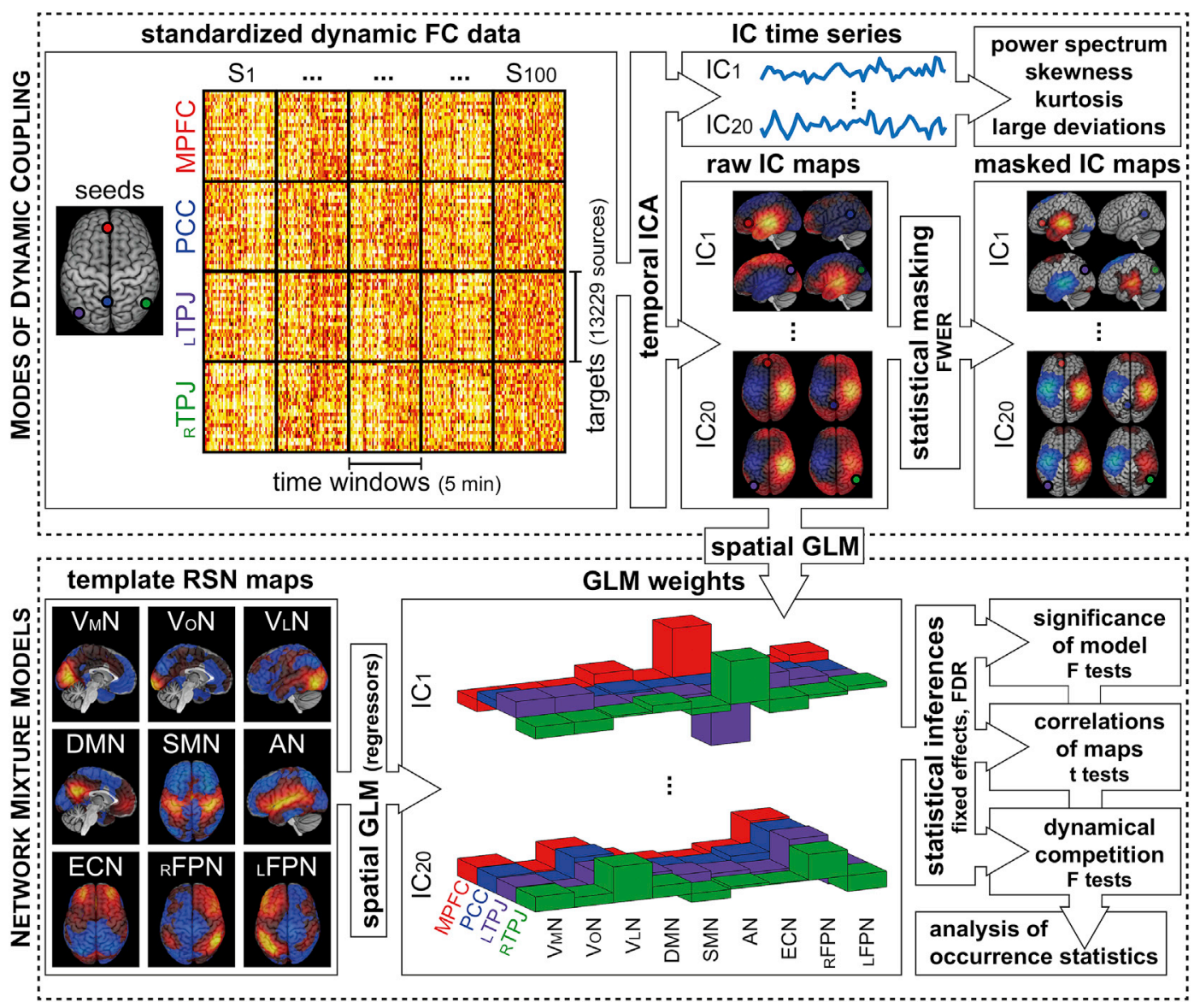

Fig. 1. Schematic illustration of the analysis pipeline. (Example for the dynamic integration of the DMN) Top: Identifying modes of dynamic coupling. The standardized seed-based FC time series are concatenated temporally across subjects $\left(S_{1}, S_{2}, \ldots, S_{100}\right)$ and spatially across seeds (indicated by colored discs in the left insert) and then submitted to a temporal ICA. This outputs several IC time series, whose power spectrum, higher-order temporal statistics, and large-deviation events are then analyzed, as well as associated IC maps (one per seed), which are masked statistically. Bottom: Network mixture modeling. Nine template RSN maps are used as regressors for a spatial GLM applied to each raw (unmasked) IC map. This results into a set of GLM weights defining a mixture model for each mode of dynamic coupling. Statistical inferences are then derived to establish model significance, univariate maps correlations, and dynamical competition. The significance of detection rates across modes of dynamic coupling is further quantified using occurrence statistics.

$20 \times 9 \times 4$ ICs) between this tail probability and the kurtosis. This approach led to a threshold of 2.5 . To assess the recurrent character of IC large deviations and their tendency to happen in isolation (notwithstanding the temporal independence constraint of ICA that penalizes simultaneous events, see also Supplementary Methods S1), we also estimated the distribution of (co-)occurrences, i.e., the number of events identified in each time window.

The effects of the frequency band $\left(\theta, \alpha, \beta, \beta^{\prime}\right)$ and seed RSN (Table 1 ) on the distributions across modes of the IC kurtosis, the IC events lifetime, and the volume fraction of IC masks were assessed using a nonparametric two-way ANOVA (Friedman test) and post-hoc Wilcoxon rank tests.

\subsection{Network mixture modeling}

To identify modes of dynamic coupling that involve cross-network integration between RSNs, we sought to establish a relationship between each raw IC map and a mixture of one or more static RSN maps.

Template RSN maps were derived from ten standard RSNs identified via a twenty-component ICA of fMRI resting-state data available at https ://www.fmrib.ox.ac.uk/datasets/brainmap+rsns (Smith et al., 2009). These fMRI maps were smoothed with a Gaussian kernel of $8 \mathrm{~mm}$ full-width-at-half-maximum using SPM8 (Friston et al., 2007) and resampled to the MEG cortical grid to obtain same size and comparable spatial smoothness across the two modalities. Of notice, the cerebellar
RSN of Smith et al. (2009) was discarded since it does not involve cortical areas, resulting in nine template RSN maps: the visuo-medial (VMN), visuo-occipital (VoN), and visuo-lateral ( $\mathrm{VLN}_{\mathrm{LN}}$ ) networks, the DMN, the SMN, the AN, the executive-control network (ECN), and the R/LFPN (see the template RSN maps in Fig. 1).

We then used general linear modeling (GLM) with these template RSN maps as regressors (bottom part of Fig. 1). This analysis differs from the standard use of GLM in neuroimaging (typically established voxel by voxel using sampling over subjects or time, see, e.g., Friston et al., 1994) by the fact that sampling is here over the cortical sources. Estimation of the GLM weights followed a standard procedure without regularization since the resulting design matrix was full rank (see Supplementary Methods S4). Statistical inference was based on our assessment of the effective number of spatial degrees of freedom in MNE source maps, i.e., $\rho=46$. This setup takes into account the autocorrelation structure due to spatial leakage (Wens et al., 2015) and thus avoids wrongly inflating significance (see, e.g., Monti, 2011, for a review of the consequences of sample autocorrelation for GLMs in the context of fMRI time series).

The network mixture model of a mode of dynamic coupling was then defined as the set of GLM weights associated with the $S$ seed nodes and the nine template RSNs (see the GLM weights in Fig. 1). For each mode and seed, model significance was established using $F$ tests (Friston et al., 2007) at $p<0.05$ with false discovery rate (FDR) correction to control for multiple comparisons (i.e., 20 modes $\times S$ seeds). Modes associated with significant mixture models for at least one seed were interpreted as 
indicative of a cross-network interaction. To obtain a clearer picture of which RSNs are involved in significant mixtures, we also investigated post hoc the univariate (partial) correlations between each raw IC map and each template RSN map via two-tailed $t$ tests (Friston et al., 2007) at $p<0.05$ corrected for the same amount of multiple comparisons as done for the $F$ tests. All statistical inferences were based on a fixed-effect design since the regressors were built upon deterministic template RSN maps.

Full details on this spatial GLM and the associated statistical tests are provided in the Supplementary Methods S4.

\subsection{Dynamical competition testing}

We also used the framework of network mixture modeling to investigate the idea that different RSNs "compete dynamically" to establish functional connections. Based on our discussion above about the relative sign of IC maps, we reasoned that such competitive behavior must involve substantial temporal anticorrelations among the cross-network couplings identified in a given mode of dynamic coupling. This situation is reflected by the presence of both positive and negative weights in a mixture model. To investigate formally the existence of dynamical competition in this sense, we thus compared statistically each network mixture model to a "non-competitive" model built under the constraint that all GLM weights have the same sign (i.e., weights are all positive or all negative), which idealizes the absence of competition. Importantly, imposing the same sign across the seeds considered (Table 1) was necessary to include the effect of relative sign across the IC maps and detect at once any instance of competition (i.e., among RSNs for fixed seed, among seeds for fixed template RSN, or between different seed-RSN pairs). Further background is provided in the Supplementary Methods S5 and 56.

We derived the exact solution of these sign-constrained GLMs using a semi-analytical approach detailed in the Supplementary Methods S7. Our technique combines (i) the observation that the sign-constrained problem corresponds to an ordinary, analytically solvable GLM under certain (but a priori unknown) zero-weight constraints, with (ii) a numerical approximation of the solution via a projected gradient descent algorithm (whose convergence can be analyzed using fixed-point methods; see, e.g., Jung, 2017) to determine the zero-weight constraints.

For each mode and seed, we then assessed statistically whether the original (unconstrained) GLM provided a significantly better model than the sign-constrained GLM. The model comparison was performed using $F$ tests (at $p<0.05$ corrected for the same amount of multiple comparisons as done for the model assessment $F$ tests) since the two GLMs are nested (Friston et al., 2007). Modes associated with significantly better unconstrained GLM for at least one seed were deemed to exhibit significant RSN competition. The only exception to this claim was when the unconstrained GLM did satisfy the sign condition since the associated $F$ statistic was then ill-defined. In this case, however, the mode was clearly non-competitive.

The technical details are developed fully in the Supplementary Methods S4, S7, and S8.

\subsection{Occurrence of cross-network integration and competition}

We quantified the tendency of modes to be structured into crossnetwork interactions, to target specific RSNs, and to exhibit dynamical competition. The occurrence rate for cross-network coupling was estimated as the proportion of modes with a significant network mixture model, that for RSN detection, as the proportion of significant mixture models with a significant correlation $t$ test with the corresponding template RSN for at least one seed, and that for dynamical competition, as the proportion of significant mixture models with significant model comparison $F$ test for at least one seed. Significance testing for these proportions was based on the binomial distribution associated with the expected false positive rate (i.e., the FDR-corrected critical $p$ value) of these tests. We report one-tailed significance at $p<0.05$ with Bonferroni correction, but also consider uncorrected significance in cases of small sample sizes where the sensitivity of these statistics is limited (see, e.g., Krzywinski and Altman, 2014).

We further applied Friedman and Wilcoxon tests to identify possible effects of frequency band or seed RSN on the proportion of cross-network coupling occurrence.

\section{Results}

To examine temporal synchrony among the cortical interactions of each RSN (Table 1), we used an ICA that decomposed the band-limited FC data into twenty modes of dynamic coupling. Each mode was associated with a number of IC maps that locate synchronously varying couplings with the seed nodes of the RSN under consideration (Table 1) and one IC time series encoding their temporal dynamics. Their spatial and temporal signatures were then analyzed statistically as outlined in Fig. 1.

To ease the understanding and interpretation of the quantitative results, we start by describing qualitatively two example modes obtained from the $\alpha$-band FC data of the DMN. We then consider the distribution of key spatio-temporal characteristics of the modes across all RSNs and frequency bands. An analysis of the modes is developed with more detail in the Supplementary Results S2 for the case of the $\alpha$ - and the $\beta$-band DMN.

\subsection{Example modes of dynamic coupling}

Figure 2 (left) summarizes the characteristics of the mode $\alpha_{2}$ (i.e., the $2^{\text {nd }}$ IC obtained in the $\alpha$ band) for the DMN. The positive part of its raw IC maps (Fig. 2, left, top) was strongest over the occipital, the occipitotemporal, and the posterior parietal cortices bilaterally and also peaked over the pre-frontal area. Their negative part covered the central sulci bilaterally. These FC topographies could also be explained in terms of cross DMN-RSN integration using network mixture modeling. The GLM weights (Fig. 2, left, bottom left) disclosed a positive contribution of visual networks (VNs) - most prominently the $\mathrm{V}_{\mathrm{LN}}$-and of the DMN (cross DMN-VN and intra-DMN couplings) mainly, and a negative contribution of the SMN (cross DMN-SMN coupling). This corresponds to a situation where cross DMN-SMN integration competes with intra-DMN and DMNVN integration. The sign of the IC maps and their GLM weights could be interpreted by considering the skewness of the IC time series, which was positive (see the arrow on the maps scale in Fig. 2, left). This means that the mode $\alpha_{2}$ is associated with increases of cross DMN-VN and intra-DMN couplings and concomitant decreases of cross DMN-SMN couplings, rather than the opposite. Further analysis of the IC time series showed that these FC modulations were non-periodic and occurred as recurrent, sporadic transient events (Fig. 2, left, bottom right).

As a second example, we consider the DMN mode $\alpha_{20}$ (Fig. 2, right). Spatially, the SMN emerged clearly from the four IC maps but with opposite signs in the left and the right hemispheres. Temporally, this mode also exhibited aperiodicity, transience, and recurrence, but now IC skewness was close to zero (indicated by a double-headed arrow on the maps scale in Fig. 2, right). The mode $\alpha_{20}$ thus corresponds to a nonperiodic, competitive mode of cross DMN-SMN integration wherein transient events of increased coupling with the right-hemispheric part of the SMN and decreased coupling with the left-hemispheric part alternate with events of decreased coupling with the right-hemispheric SMN and increased coupling with the left-hemispheric part. However, network mixture modeling failed to detect this cross DMN-SMN integration. In fact, this example illustrates the two caveats of this approach. First, the GLM weights corresponding to the SMN were close to zero because the IC maps asymmetry destroyed any correlation with the full SMN map. Generally, the sensitivity to template RSN maps identification is lessened in cases where the target RSN is split into sub-networks. Second, the GLM 

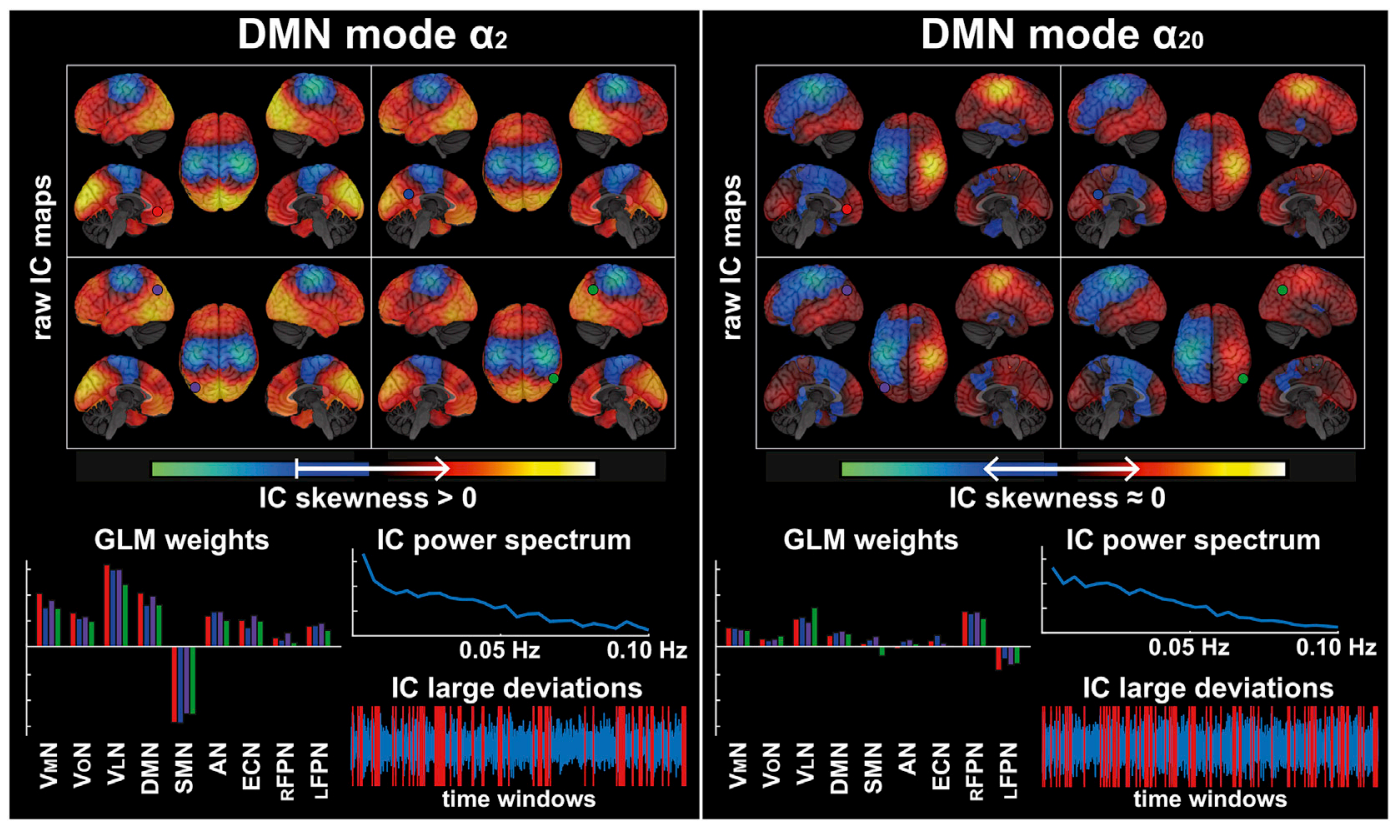

Fig. 2. Example modes of dynamic coupling. Qualitative characteristics are shown for two ICs of the DMN-based FC data in the $\alpha$ band, here without statistical assessment. Spatial aspects encompass the four raw IC maps (top) and the associated network mixture model weights (bottom left). Seeds are color coded (red: MPFC, blue: PCC, violet: LTPJ, green: RTPJ). Temporal properties include the skewness (sign of non-zero skewness indicated by a single arrow on maps scale, approximately zero skewness indicated by a double-headed arrow), the kurtosis and the corresponding large-deviation events (emphasized in red on the IC time course), and the power spectrum (bottom right) of the IC time series. Scales and units are set consistently across the two modes but are otherwise arbitrary.

weights associated with the FPNs emerged with opposite signs because the SMN peaks leaked towards the frontal and the posterior parietal cortices, leading to a spurious interpretation of the mode in terms of alternating cross DMN-R/LFPN integration. This is due to the intrinsic blurriness of MEG-based FC mapping that persists after leakage correction (see, e.g., Wens et al., 2015) and limits the specificity of RSN identification.

\subsection{Temporal dynamics of the modes}

The aperiodicity and the transient character of IC time series observed in the two preceding examples (Fig. 2) generalized to all the modes. The IC skewness was significant for about half of the modes and the IC kurtosis, for all of them. The latter result confirms that each mode exhibited large deviations. Further, comparing the distributions of IC kurtosis across modes obtained with different RSNs and frequency bands (Fig. 3, top left), we identified an effect of the band ( $p=2.2 \times 10^{-19}$, Friedman test) but not of the RSN ( $p=0.09)$. The band effect was due to a larger median across the $\alpha$-band modes, indicating that some of these modes exhibited higher IC kurtosis than in the other bands, irrespectively of the seed RSN.

The transience of the large-deviation events (implied by high IC kurtosis) was confirmed by considering their lifetime distribution (Fig. 3, bottom left), whose median was only slightly above the windows step size. We observed a band effect $\left(p=2.3 \times 10^{-7}\right)$ without RSN effect ( $p=$ 0.49 ) reflecting a tendency for longer-lived events in the $\alpha$ band and for shorter-lived events in the $\beta$ band. The latter was explained by the fact that no $\beta$-band mode exhibited lifetimes above $6.4 \mathrm{~s}$, whereas longer lifetimes emerged in the other bands (maximum lifetime across $\theta$-band modes: $7.3 \mathrm{~s}, \alpha: 7.5 \mathrm{~s}, \beta^{\prime}: 6.9 \mathrm{~s}$ ). The former was explained by a higher number of $\alpha$-band modes with lifetimes above those of the $\beta$ band (proportion across $\theta$-band modes: $5 \%, \alpha: 8 \%, \beta$ : $1 \%$ ). This is illustrated explicitly in the Supplementary Results S3.

Table 2 shows that large-deviation events occur fairly often, since $35 \%$ of the time windows contained at least one event. Simultaneous occurrences are scarce, as $77 \%$ of large deviations were isolated and $20 \%$ consisted of two simultaneous events. The co-occurrence of more than two events turned out to be rare (about $3 \%$ of the time windows).

Finally, we note that the combination of higher kurtosis and longer lifetimes for $\alpha$-band modes also implicates a tendency for larger deviations (see Supplementary Results S3).

\subsection{Spatial organization of the modes}

We now consider the spatial structure of the modes of dynamic coupling (notwithstanding the specificity/sensitivity issues identified above). All IC maps involved significant contributions to the FC data. Further, they all exhibited positive and negative significant values, which indicates that coupling synchronization is typically accompanied with coupling anticorrelation. We assessed the spatial extent of the significant FC patterns associated to each mode by measuring the fraction of volume filled by the IC statistical masks (Fig. 3, top right). Comparing their distributions showed that the DMN modes tended to be more focused spatially (RSN effect, $p=2.1 \times 10^{-8}$ ) and that the $\beta$-band modes tended to be more extended (band effect, $p=3.2 \times 10^{-10}$ ).

We also determined whether these FC patterns could be understood in terms of cross-network integration using network mixture modeling. The detection rate of significant mixtures was higher than expected by chance in all cases ( $p<10^{-3}$, binomial tests) except for the VN and the RFPN in the $\theta$ band $(p>0.04)$. This means that most FC datasets revealed some cross-RSN interactions. This occurrence rate appeared substantial for the DMN, where a large majority of modes could be classified in terms of cross-network coupling patterns, but smaller for the other RSNs (Fig. 3, bottom right). This was confirmed statistically by a RSN effect on these proportions ( $p=2.9 \times 10^{-18}$, Friedman test). We also disclosed a band effect $\left(p=5.7 \times 10^{-4}\right.$ ) due to higher occurrence rates in the $\alpha$ and the $\beta$ bands compared to the $\theta$ and the $\beta^{\prime}$ bands. Note in Fig. 3 (bottom right) that the comparison for the $\beta$ and the $\beta^{\prime}$ bands was only barely nonsignificant ( $p=0.06$, Wilcoxon test).

We further sought to identify which RSNs were predominantly 

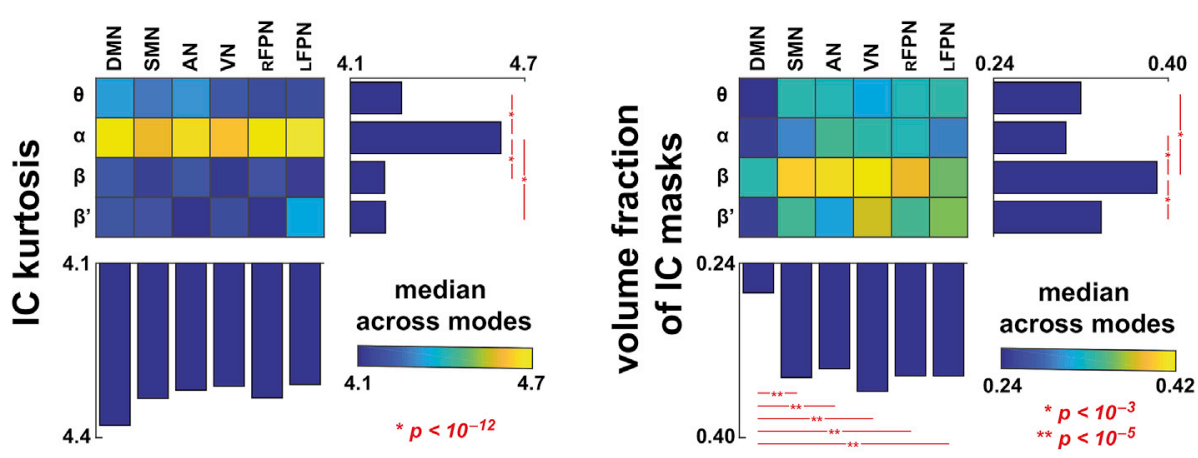

Fig. 3. Effects of seed RSN and frequency band on characteristics of the modes of dynamic coupling. The distributions across modes of the IC kurtosis (top left), the IC large-deviation events lifetime (bottom left), the volume fraction of IC statistical masks (top right), and the proportion of significant network mixture models (bottom right) are compared across frequency bands $\left(\theta, \alpha, \beta, \beta^{\prime}\right)$ and seed RSNs (see Table 1). The bar plots show the median values computed over RSNs (right side) or bands (bottom side). Significant effects identified via post-hoc Wilcoxon rank tests are indicated on these bar plots by red stars.
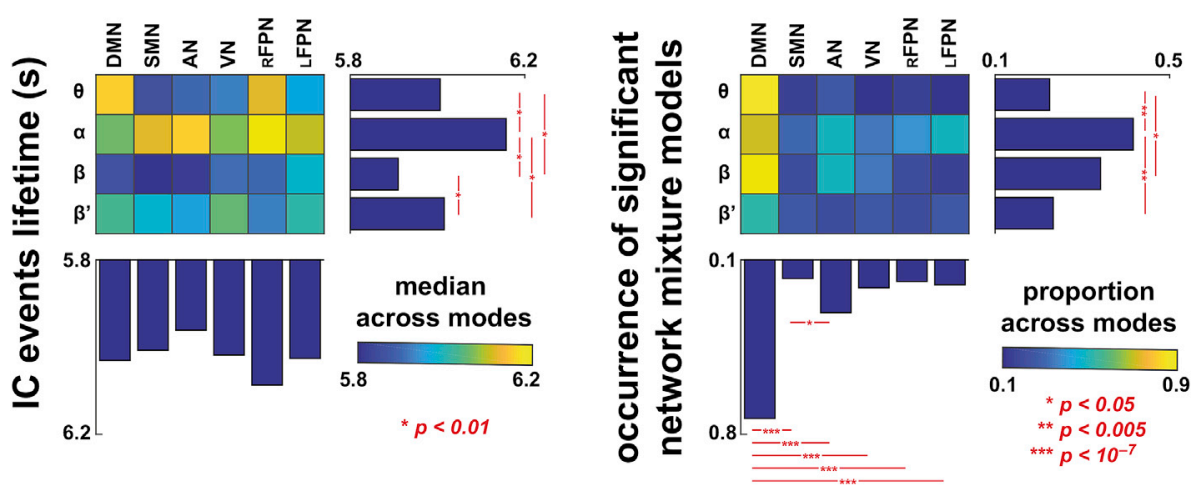

involved among the cross-network couplings obtained in mixture models (Fig. 4, top). For the DMN modes, we detected a wide range of cross DMN-RSN interactions more often than expected by chance, particularly in the $\beta$ band where RSN occurrence was significant for all the template RSNs. Interestingly, cross-network integration appeared more restricted in the $\alpha$ band where only the DMN-VMN, DMN-VLN, and DMN-SMN couplings were detected with significant rate. Patterns of RSN occurrence were comparably scarcer with the other seed RSNs (in line with Fig. 3, bottom right). It is noteworthy that the VoN occurred significantly often only among the DMN modes in the $\beta$ band, and not at all in the other cases (bar a non-significant occurrence for the $\beta^{\prime}$-band DMN).

\subsection{Competition among cross-network couplings}

The detection rates for dynamical competition among the significant mixture models are shown in Fig. 4 (bottom). A significant majority of DMN modes exhibited competitive cross-network couplings, with the highest occurrence rates among the $\alpha$ - and the $\beta$-band modes. Competition also emerged with the other seed RSNs, although significance was more variant. This could merely reflect the scarcer occurrence of significant mixture models in these cases (Fig. 3, bottom right), which limits statistical power. No competition was detected for the VN modes in the $\theta$ band and for the LFPN modes in the $\theta$ and the $\beta$ bands, and the detection rate for the $\alpha$-band LFPN was below the uncorrected significance level.

Interestingly, this widespread detection of dynamical competition contrasts with a similar analysis performed at the level of static FC, where no significant RSN map appeared to display RSN competition (see Supplementary Results S1).

Table 2

Probability distribution $P_{n}$ that $n$ large-deviation events occur simultaneously. The probability was estimated by pooling (co-)occurrence counts across all frequency bands $\left(\theta, \alpha, \beta, \beta^{\prime}\right)$ and all RSNs (see Table 1$)$. The counting process is illustrated in the Supplementary Results S3.

\begin{tabular}{lllllllll}
\hline$n$ & 0 & 1 & 2 & 3 & 4 & 5 & 6 & $\geq 7$ \\
\hline$P_{n}(\%)$ & 65 & 27 & 7 & 1 & 0.2 & 0.01 & 0.003 & 0 \\
\hline
\end{tabular}

\section{Discussion}

\subsection{Summary of findings}

This paper investigates spontaneous synchronization patterns of dynamic FC with RSN nodes, or in short, modes of dynamic coupling. These modes corresponded to recurrent and sporadic transient events of FC changes involving varied and widespread coupling patterns. The $\alpha$ band disclosed longer lived FC events with larger deviations. The $\beta$-band modes involved spatially more extended coupling patterns. Crossnetwork integration was predominant among the DMN modes but scarcer among the others, and it was more common in the $\alpha$ and the $\beta$ bands. The dynamic integration of the DMN with other RSNs involved mostly the SMN and the $\mathrm{V}_{\mathrm{L}} / \mathrm{MN}$ in the $\alpha$ band, most RSNs in the $\theta$ and the $\beta^{\prime}$ bands, and all of them in the $\beta$ band. Importantly, and in contradistinction with static FC, dynamical competition among these cross DMNRSN couplings was ubiquitous.

\subsection{Resting-state dynamics and coupling synchrony}

While the nature and function of spontaneous brain interactions remain difficult to investigate experimentally, the picture emerging from empirical FC studies is one of a dynamic integration involving transient RSN fragmentation into sub-networks, cross-network binding, and alternation of core hubs (for reviews, see, e.g., Hutchison et al., 2013; de Pasquale et al., 2018). This entails a resting-state dynamics that has been mainly conceptualized as a jump process between discrete, temporally non-overlapping FC states that recur at random times. Two notions of FC states have been devised: transiently synchronized networks at the supra-second timescale (Allen et al., 2014; O'Neill et al., 2015b) and sub-second states detectable using hidden Markov models (Baker et al., 2014; Vidaurre et al., 2018). The latter have been suggested to underlie the former (Baker et al., 2014), however they are inaccessible to sliding-window FC and will thus not be discussed in detail hereafter. The supra-second jump dynamics is also compatible with the observation (within periods of concomitant strong intra-RSN coupling and high RSN centrality) of a few core networks playing in alternation, and swiftly 

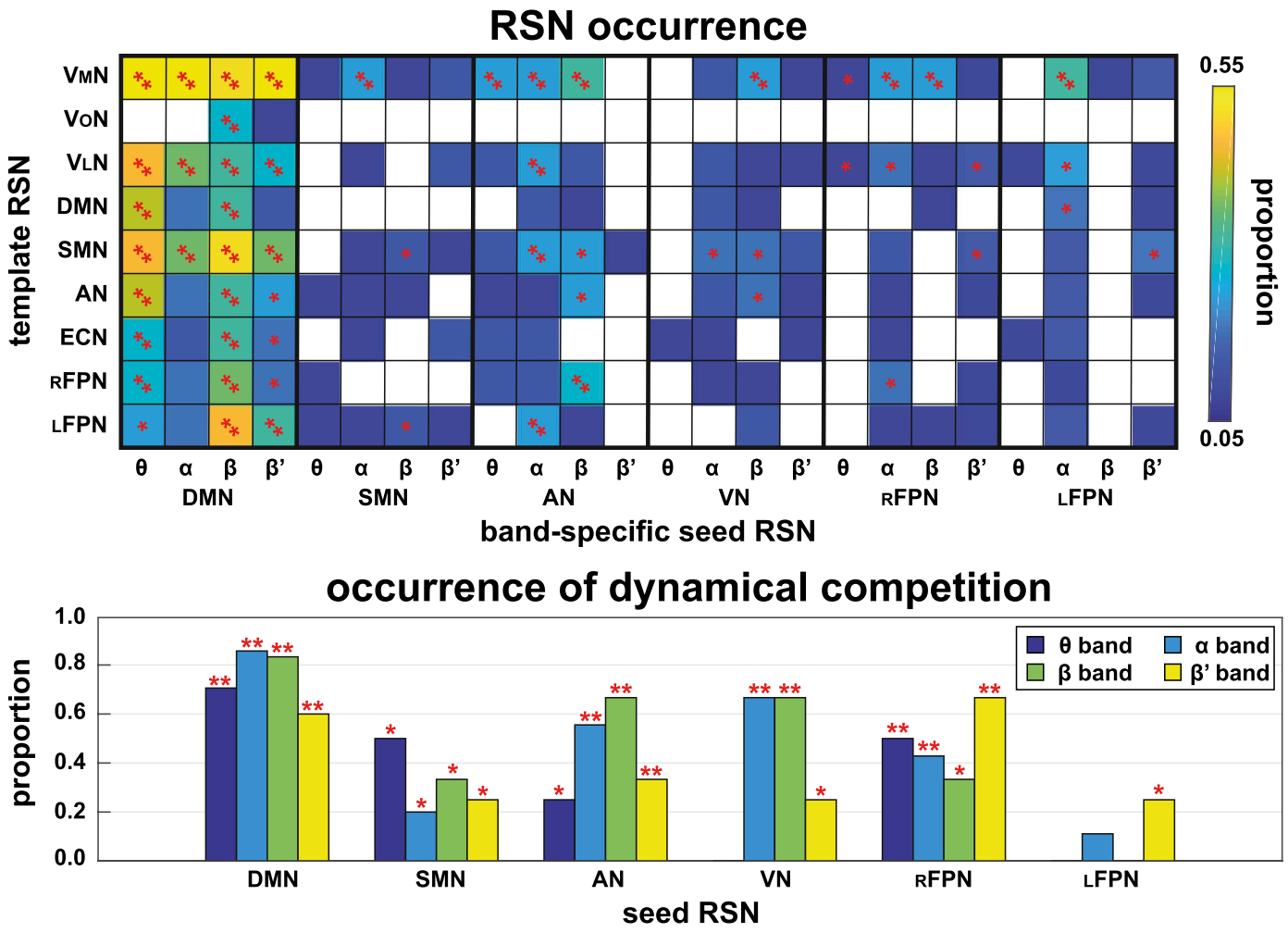

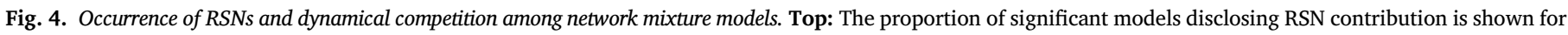

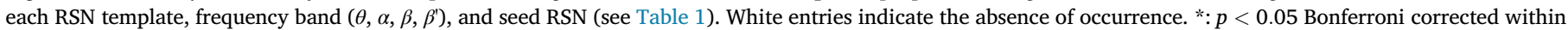

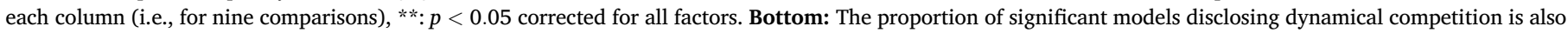
shown for each band and seed RSN. *: $p<0.05$ uncorrected, $* *: p<0.05$ Bonferroni corrected. Significance of occurrence rates is based on binomial tests.

shifting, the role of global brain integrator (de Pasquale et al., 2012, 2016).

The concept of coupling synchrony differs from these aspects and thus reveals novel properties of dynamic functional integration, but it is also compatible with the existing picture. We make four general inferences about resting-state dynamics:

(i) Spontaneous brain activity is characterized by coordinated coupling fluctuations around its RSN backbone.

(ii) These synchronized couplings reflect transition events between transiently synchronized networks.

(iii) Coupling synchronization is transient and covers large cortical areas, especially in the $\beta$ band.

(iv) Events of coupling synchronization outside the $\beta$ band can be longer lived, especially in the $\alpha$ band.

Inference (i) is based on the result that all modes of dynamic coupling disclosed significant IC maps, i.e., they explained a significant fraction of FC variance. This reveals that not only is resting-state activity structured into RSNs, but its functional interactions themselves are spontaneously organized into modes, which may thus be viewed by analogy as "networks of couplings" (O'Neill et al., 2015a). State analysis (Allen et al., 2014; O'Neill et al., 2015b) could not lead directly to this observation since it is geared towards the detection of stable patterns rather than their transition per se (because of the strict temporal exclusion constraint).

Inference (ii) asserts that the coupling synchrony we uncovered relates specifically to these FC state transitions. This is inevitable if we take state models literally (since FC changes can only occur at state jumps), but then they represent a simplification of the underlying neural dynamics. Empirically too, the spatio-temporal characterization of our modes depicts them as transition processes between successive transiently synchronized networks. Indeed, all the modes corresponded to transient events of FC changes that were recurrent, aperiodic, and mostly isolated, which fits with random state jumps (Allen et al., 2014). They also involved both increases and decreases as may be expected for transitions reflecting the difference between two successive states. Furthermore, in this context, the modes associated with preserved FC change sign can be interpreted as encoding non-reversible state transitions (i.e., they are more likely to occur in one direction than its opposite) and those with alternating sign, as reversible transitions. Therefore, our results are not only compatible with the jump process theory of resting-state dynamics but actually complements it. Still, this claim should be taken with two provisos. First, our confirmation is not completely unbiased because our ICA seeked transient, temporally sparse dynamics (see Calhoun et al., 2013; Daubechies et al., 2009, for a discussion of this point in the context of fMRI). This bias alone cannot explain the strongly transient character of our modes (see below and Supplementary Methods S2) but, critically, it may render our analysis insensitive to possible modes of dynamic coupling associated with less prominent temporal sparsity. Second, further study is required to prove the link between modes of dynamic coupling and FC state transitions.

The association between modes of dynamic coupling and transient FC events can also be validated with simulations. In the Supplementary Results S4, we used synthetic MEG data generated from a simple two-state system and identified a single mode corresponding to their transition. It also fits well within the framework of Deco et al. (2011) based on large-scale neurocomputational models of the human connectome. Their simulations suggested that resting-state activity is generated by a near-critical, multistable system composed of attractors corresponding to network configurations tightly constrained by anatomy but destabilized by local, stochastic or chaotic fluctuations within neural populations (Deco et al., 2009; Deco and Jirsa, 2012; Hansen et al., 2015). This generates a metastable dynamics characterized by fast transitions between attractors, which we suggest are captured empirically by the modes of dynamic coupling (the stable periods in between transitions being presumably encoded in FC states, see, e.g., Hutchison et al., 2013). Besides, our analysis 
indicates a mixture of reversible and non-reversible processes. It would be interesting to confront this finding with computational models and see if (non)reversibility is an emergent property of the human connectome.

Inference (iii) refers to the fact that the modes of dynamic coupling involved FC events that are short lived (less than $10 \mathrm{~s}$ ) and cover a substantial fraction of the cortex, and significantly more so for the $\beta$-band modes. This preferential implication of $\beta$ activity agrees with its suggested role as a functional background facilitating long-range neural synchronization (Bressler and Richter, 2015; Kopell et al., 2000) and with the "statu-quo signal" theory of Engel and Fries (2010) according to which $\beta$ activity maintains the current behavioral state, e.g., the exploratory state generated by metastability (Deco et al., 2011). Accordingly, $\beta$-band FC appears to be the best (but not the only) electrophysiological correlate of this functional exploration. In fact, Deco and Corbetta (2011) proposed that this exploration ensures responsiveness to future behavioral changes by retaining active representations of possible stimuli or tasks built upon past experiences. This led to two hypotheses that have been corroborated specifically in the $\beta$ band, i.e., resting-state FC predicts task performance (Mary et al., 2015) and resembles task-positive FC in naturalistic paradigms (Betti et al., 2018). On this ground, we propose to interpret the $\beta$-band modes of dynamic coupling as reflecting the exploration at rest of complex, ecological activity patterns. Such a process would presumably require the integration of neural activity within widespread cortical areas. This also fits with the specific role played by the $\beta$ band in the theory of de Pasquale et al. (2016) whereby global brain integration is sustained dynamically by a few core networks (the DMN, the SMN, and the bilateral FPN).

The tendency of $\beta$-band modes to exhibit shorter lifetimes and wider coupling patterns does not preclude contributions from the other bands to the functional exploration generated by metastability. For instance, there is no reason to expect the emergence of $\beta$ rhythms only in the computational models of Deco et al. (2011), although it would be interesting to see if refined models (Hansen et al., 2015) could account for their special role. Further, large-deviation events were highly transient in all cases, which is indicative of metastability for the other bands as well. Actually, the fact that lifetimes were close to the lowest limit accessible to our sliding-window FC data suggests to extend our findings to finer timescales. In any case, we hypothesize that our functional interpretation above generalizes to the other bands.

Still, our inference (iv) highlights a peculiarity of the other bands and most prominently of the $\alpha$ band, namely, they exhibit modes with slightly longer lived FC events (by a couple of seconds, which is small compared to the timescale of our FC data but was sufficient to generate significant band effects). This suggests smoother state transitions that could reflect an increased stability of some FC states. This hypothesis is in line with, e.g., the proposed function of $\alpha$ rhythms for the top-down modulation of attention, perception, and consciousness level (Jensen et al., 2012; Klimesch, 2012) and that of $\theta$ rhythms for memory (Colgin, 2013; Klimesch et al., 2010). Indeed, state stabilisation occurs when the brain departs from its exploratory state and is engaged into cognitive processes (Deco and Corbetta, 2011). As attentional or perceptual drifts happen, we may thus expect certain FC states (i.e., the transiently synchronized networks that support such function) to partially stabilize. Based on this, we surmise that some non- $\beta$-band modes (specifically, those exhibiting higher kurtosis or longer lifetimes than the $\beta$-band modes) correspond to spontaneous drifts in mentation and the others, to the aforementioned functional exploration of metastable states.

That said, these hypotheses remain to be confirmed in behaviorally controlled experiments. Further, it would be interesting to generalize our analysis using a data-driven frequency band selection, as done in Vidaurre et al. (2018).

\subsection{Functional integration of the default-mode network}

So far, our interpretations of spontaneous coupling synchrony were not tied up to RSNs. The DMN stood out when we analyzed the relationship between coupling synchrony and cross-network integration. We make two inferences specifically involving the DMN:

(v) The DMN is a core network specialized in the transient integration with other RSNs.

(vi) Several RSNs compete to bind with the DMN.

Inference (v) is based on our observation that the large majority of DMN modes involved cross-network couplings. Some modes derived from the other RSNs shared this feature but many others did not. So the DMN appeared as the sole RSN that is systematically bound to other RSNs, which presumably endows it with a central role in the functional exploration generated by metastability.

This property specific to the DMN has, to our knowledge, never been emphasized. It is closely related to the core network theory of de Pasquale et al. (2016) that highlights the DMN as a global brain integrator, at least half of the time and mostly in the $\beta$ band. In fact, the alternating dynamics of core networks was partially reflected in our data. Indeed, the variety of cross DMN-RSN couplings appeared widespread among the $\beta$-band modes, with significant occurrence of all RSNs (in opposition to the $\alpha$-band modes). The highest occurrence rates across $\beta$-band modes spotted the two other core networks identified by de Pasquale et al. (2016), i.e., the SMN and the $\mathrm{R} / \mathrm{LFPN}$ (combining both FPNs, the rate for the bilateral FPN even reached 60\%), as well as the VMN. The emergence of these two core networks may be explained by the fact that their high-centrality periods must overlap (as each spends $40-50 \%$ of their time as hub, de Pasquale et al., 2016), increasing their likelihood to participate in $\beta$-band cross-network integration. On the other hand, the substantial involvement of DMN-VMN couplings and the lesser involvement of intra-DMN couplings may seem contradictory, as the VN is not part of the core networks and high DMN centrality periods overlap with strong intra-DMN FC periods (de Pasquale et al., 2016). Sill, given the difficulty with MEG to discriminate the PCC part of the DMN and the $\mathrm{VMN}_{\mathrm{N}}$, it may be that the DMN-VMN couplings reflect intra-DMN integration instead. One additional possible inconsistency is that neither the SMN nor the FPNs emerged from our data as the DMN did. Further work is needed to clarify this point.

Inference (vi) rests on our finding that a large majority of DMN modes involved a dynamical competition among cross DMN-RSN couplings. In fact, competition was not limited to the DMN and emerged with other RSNs too (although somewhat less consistently). This property fits well with the functional interpretation of metastability as a spontaneous competition among RSNs for the allocation of neural resources and optimum processing of future sensory, motor, or cognitive demands (Deco and Corbetta, 2011). Our data bring two major inputs to this hypothesis. First, this competition emerges predominantly (but not solely) at the level of DMN-RSN integration. Second, it was observable at the short timescale of dynamic FC but not in static FC (see Supplementary Results S1), which makes sense as cross-network integration is transient by nature. This last observation contrasts with fMRI studies identifying negative static FC between the DMN and the bilateral FPN (Fox et al., 2005), but they are in line with others (see, e.g., Smith et al., 2009) as well as all MEG-based RSN studies (see, e.g., de Pasquale et al., 2010, for a discussion). Actually, detecting this anticorrelation requires a global signal regression (Fox et al., 2009) that was not used here. So we cannot exclude the existence of static RSN competition, but it is presumably subtler.

Functionally speaking, competitive cross DMN-RSN integration may play a major role in the main functions of the DMN, i.e., inner-directed cognition (e.g., spontaneous thoughts, mind wandering, or the mentation of past or hypothetical events, see, e.g., Buckner et al., 2008) and conscious awareness (Baars et al., 2003; Giacino et al., 2014). In this respect, the $\alpha$-band DMN modes may be particularly informative, given their putative relation with spontaneous drifts in mentation. We speculate that the associated FC changes enable the neural communication between the DMN and other RSNs needed for, e.g., motor (DMN-SMN coupling), auditory (DMN-AN), or visual (DMN-VM/LN) imagery. It is 
noteworthy that DMN-SMN and DMN-VM/LN interactions stood out as those occurring the most among the $\alpha$-band modes, usually in competition (see Supplementary Results S2). This hints at a possible predominance at rest of a cognitive alternation between motor and visual mentations. Also of interest is the complete absence of DMN-VoN coupling, which is in line with data suggesting that the primary visual cortex is not necessary for visual imagery (de Gelder et al., 2015). This appears plausible as no direct retinal stimulation is involved, but this remains a matter of debate (see, e.g., Pearson et al., 2015).

\subsection{Methodological considerations}

Our analysis pipeline (Fig. 1) was split into two separate main steps: (i) an ICA of dynamic FC data to identify spatio-temporal patterns of synchrony among brain couplings, and (ii) network mixture modeling for their classification in terms of cross-network integration and dynamical competition.

A general issue with dynamic FC is that short-time correlations are affected by large random estimation errors, leading to difficulties in distinguishing genuine dynamics from statistical variability (Hindriks et al., 2016). One question about our ICA is thus whether the decomposition into modes was driven by FC noise rather than coupling synchronization. This was not the case because the likelihood that the distribution of excess kurtosis across our modes (which were all significantly positive) emerges from correlation errors only, was extremely small ( $p<10^{-7}$ based on numerical estimates). In fact, ICA entailed a reduction of FC noise because the modes disclosed large patterns of coupling synchrony that cannot be explained by correlation errors (which do not exhibit spatial coordination beyond the intrinsic blurriness of MEG FC), so that their contribution within each mode averaged out. These two analytical arguments are developed fully in the Supplementary Methods S2 and S3. The ability of our ICA to detect coupling synchrony among noisy dynamic FC data is also illustrated in the simulation reported in the Supplementary Results S4. Finally, note that the seminal study of O'Neill et al. (2017b) provided a proof of concept as they used a similar ICA of sliding-window correlations to identify task-related transiently synchronized networks, which appeared meaningful in view of the brain processes expected in their experimental design.

Another general confound of dynamic FC is that variations in slidingwindow correlations could be driven by transient modulations of local activity (i.e., power changes) rather than couplings per se. We show in the Supplementary Results S5 that sliding-window power estimates (with SLORETA for depth bias correction, see Pascual-Marqui, 2002) only poorly correlated with the IC time series (absolute Pearson correlations below 0.03 across all modes, RSNs, and frequency bands) and thus could not explain the spatio-temporal features of the modes. A similar analysis focusing on high-frequency power also confirmed that our results were not driven by muscle artifacts.

Yet another difficulty of our ICA of resting-state FC (i.e., without task or stimulation onset) is the subjective selection of relevant ICs, e.g., by visual inspection of their maps. Here, network mixture models allowed us to classify them objectively as linear superpositions of fMRI RSN templates. The validity of using static fMRI as reference for MEG dynamic FC is obviously debatable. The benefit is that fMRI provides clear-cut RSN atlases, which have guided both the design (de Pasquale et al., 2010, 2012; 2016; Sockeel et al., 2016) and the interpretation (Brookes et al., 2011; Liu et al., 2017) of MEG/EEG FC analyses. Resting-state fMRI atlases are also used as functional brain parcellations in MEG studies (see, e.g., Vidaurre et al., 2018). The standard and reproductible character of fMRI RSNs is a crucial aspect because the ensuing interpretations are tied up to the choice of templates. Of note, the RSN atlas of Smith et al. (2009) miss a couple of known systems, e.g., the ventral attentional and the language networks. This might $a$ priori incur a lack of classification sensitivity for these two RSNs, but this is presumably mitigated by the fact that they have been scarcely disclosed with MEG resting-state FC. A more fundamental drawback lies in the different nature of the signals, the distinct spatial and temporal resolutions, and in the usage of static RSNs. So our mixture models should not be over-interpreted as genuine models of dynamic integration. They merely provide a crude approximation, but one that still allowed detection of several cross-network coupling patterns. We reported a lessened sensitivity to patterns involving sub-networks (our prototype was the mode $\alpha_{20}$, Fig. 2 right) but this issue appeared relatively limited and only a few modes were totally misclassified (see Supplementary Results S2). This is because spatial correlation between a full RSN and a sub-network map may be sensitive to their partial overlap. We also noted a restricted specificity related to the intrinsic blurriness of MEG FC rather than to the use of fMRI templates.

Technically, we constructed the mixture models using GLM weights, which provide a multivariate measure of maps correlations. However, their values must be interpreted cautiously. First, effect sizes are strongly inflated if maps autocorrelation (dominated by MEG spatial leakage) is not controlled. That is why we considered standardized weights wherein the reduction in spatial degrees of freedom was built in (see Supplementary Methods S4). Second, cross-correlations among GLM regressors lead to various suppression effects, which are beneficial to the GLM as a whole but may render individual weight values ambiguous (Watson et al., 2013). Specifically, cross-over suppression entails a magnitude boost of some weights while dampening or even reversing the sign of others (Watson et al., 2013). In particular, the sign-reversal possibility challenges the validity of our novel dynamical competition test. However, there is no such issue in our case because the RSN templates were weakly correlated (absolute value of pairwise Pearson correlations: $0.04 \pm 0.02$ ) so our GLM design was well conditioned (see Supplementary Methods S5 and Supplementary Results S6 for full justification). Actually, this provides another argument for using a fMRI-based RSN atlas in our mixture models. Last, we emphasize that GLMs do not provide causal information (Weichwald et al., 2015). So mixture models cannot inform us on whether superpositions of static RSNs underlie modes of dynamic coupling, whether these modes generate RSNs, or whether both have a common factor (e.g., hidden brain states or metastable attractors).

Despite its limitations, some of which may hopefully be overcome in future developments, our current approach allowed to uncover new features of the metastable resting-state dynamics and the specialized function of the DMN for dynamic cross-network integration. It could also come in handy to reveal the impact of behavioral manipulations or brain disorders on the intrinsic functional organization of the human brain.

\section{Acknowledgements}

This work was supported by the Action de Recherche Concertée (ARC Consolidation 2015-2019, "Characterization of the electrophysiological bases, the temporal dynamics and the functional relevance of resting state network" attributed to X.D.T.) and by the research convention "Les Voies du Savoir" (Fonds Erasme, Brussels, Belgium). M.B. benefited from the program Attract of Innoviris (grant 2015-BB2B-10), the Spanish Ministry of Economy and Competitiveness (grant PSI2016-77175-P), and the Marie Skłodowska-Curie Action of the European Commission (grant 743562). M.V.G. and G.N. were supported by the Fonds Erasme. N.C. benefited from a research grant from the ARC Consolidation (2014-2017, "Characterization of the electrophysiological bases, the temporal dynamics and the functional relevance of resting state network" attributed to X.D.T.) and from the Fonds Erasme (research convention "Les Voies du Savoir"). X.D.T. is Post-doctorate Clinical Master Specialist at the Fonds de la Recherche Scientifique (F.R.S.-FNRS, Brussels, Belgium). The MEG project at the CUB - Hôpital Erasme is financially supported by the Fonds Erasme (research convention "Les Voies du Savoir").

\section{Appendix A. Supplementary data}

Supplementary data to this article can be found online at https://doi. $\operatorname{org} / 10.1016 /$ j.neuroimage.2019.05.081. 


\section{References}

Allen, E.A., Damaraju, E., Plis, S.M., Erhardt, E.B., Eichele, T., Calhoun, V.D., 2014. Tracking whole-brain connectivity dynamics in the resting state. Cerebr. Cortex 24 663-676.

Baars, B.J., Ramsoy, T.Z., Laureys, S., 2003. Brain, conscious experience and the observing self. Trends Neurosci. 26, 671-675.

Baker, A.P., Brookes, M.J., Rezek, I.A., Smith, S.M., Behrens, T., Probert Smith, P.J., Woolrich, M.W., 2014. Fast transient networks in spontaneous human brain activity. Elife 3, e01867.

Bastos, A.M., Schoffelen, J.M., 2015. A tutorial review of functional connectivity analysis methods and their interpretational pitfalls. Front. Syst. Neurosci. 9, 175.

Beckmann, C.F., DeLuca, M., Devlin, J.T., Smith, S., 2005. Investigations into resting-state connectivity using independent component analysis. Philos. Trans. R. Soc. Lond. B Biol. Sci. 360, 1001-1013.

Betti, V., Corbetta, M., de Pasquale, F., Wens, V., Della Penna, S., 2018. Topology of functional connectivity and hub dynamics in the beta band as temporal prior for natural vision in the human brain. J. Neurosci. 38 (15), 3858-3871.

Biswal, B., Yetkin, F.Z., Haughton, V.M., Hyde, J.S., 1995. Functional connectivity in the motor cortex of resting human brain using echo-planar MRI. Magn. Reson. Med. 34 $537-541$.

Bourguignon, M., De Tiège, X., Op de beeck, M., Ligot, N., Paquier, P., Van Bogaert, P., Goldman, S., Hari, R., Jousmäki, V., 2013. The pace of prosodic phrasing couples the listener's cortex to the reader's voice. Hum. Brain Mapp. 34, 314-326.

Bourguignon, M., De Tiège, X., Op de beeck, M., Pirotte, B., Van Bogaert, P., Goldman, S., Hari, R., Jousmäki, V., 2011. Functional motor-cortex mapping using corticokinematic coherence. Neuroimage 55, 1475-1479.

Bressler, S.L., Richter, C.G., 2015. Interareal oscillatory synchronization in top-down neocortical processing. Curr. Opin. Neurobiol. 31, 62-66.

Brookes, M.J., Liddle, E.B., Hale, J.R., Woolrich, M.W., Luckhoo, H., Liddle, P.F., Morris, P.G., 2012a. Task induced modulation of neural oscillations in electrophysiological brain networks. Neuroimage 63, 1918-1930.

Brookes, M.J., O'Neill, G.C., Hall, E.L., Woolrich, M.W., Baker, A., Corner, S.P., SaE, R., Morris, P.G., Barnes, G.R., 2014. Measuring temporal, spectral and spatial changes in electrophysiological brain network connectivity. Neuroimage 91, 282-299.

Brookes, M.J., Woolrich, M., Luckhoo, H., Price, D., Hale, J.R., Stephenson, M.C., Barnes, G.R., Smith, S.M., Morris, P.G., 2011. Investigating the electrophysiological basis of resting state networks using magnetoencephalography. Proc. Natl. Acad. Sci. U. S. A. 108, 16783-16788.

Brookes, M.J., Woolrich, M.W., Barnes, G.R., 2012b. Measuring functional connectivity in MEG: a multivariate approach insensitive to linear source leakage. Neuroimage 63, 910-920.

Buckner, R.L., Andrews-Hanna, J.R., Schacter, D. 1., 2008. The brain's default network: anatomy, function, and relevance to disease. Ann. N. Y. Acad. Sci. 1124, 1-38.

Calhoun, V.D., Potluru, V.K., Phlypo, R., Silva, R.F., Pearlmutter, B.A., Caprihan, A., Plis, S.M., Adali, T., 2013. Independent component analysis for brain FMRI does indeed select for maximal independence. PLoS One 8, e73309.

Chang, C., Glover, G.H., 2010. Time-frequency dynamics of resting-state brain connectivity measured with fmri. Neuroimage 50, 81-98.

Clumeck, C., Suarez Garcia, S., Bourguignon, M., Wens, V., Op de Beeck, M., Marty, B., Deconinck, N., Soncarrieu, M.V., Goldman, S., Jousmäki, V., Van Bogaert, P., De Tiège, X., 2014. Preserved coupling between the reader's voice and the listener's cortical activity in autism spectrum disorders. PLoS One 9, e92329.

Colgin, L.L., 2013. Mechanisms and functions of theta rhythms. Annu. Rev. Neurosci. 36 (1), 295-312.

Damoiseaux, J.S., Rombouts, S.A., Barkhof, F., Scheltens, P., Stam, C.J., Smith, S.M., Beckmann, C.F., 2006. Consistent resting-state networks across healthy subjects. Proc. Natl. Acad. Sci. U. S. A. 103, 13848-13853.

Daubechies, I., Roussos, E., Takerkart, S., Benharrosh, M., Golden, C., D'Ardenne, K., Richter, W., Cohen, J.D., Haxby, J., 2009. Independent component analysis for brain fMRI does not select for independence. Proc. Natl. Acad. Sci. U. S. A. 106, 10415-10422.

de Gelder, B., Tamietto, M., Pegna, A.J., Van den Stock, J., 2015. Visual imagery influences brain responses to visual stimulation in bilateral cortical blindness. Cortex 72, 15-26.

de Pasquale, F., Corbetta, M., Betti, V., Della Penna, S., 2018. Cortical cores in network dynamics. Neuroimage 180, 370-382.

de Pasquale, F., Della Penna, S., Snyder, A.Z., Lewis, C., Mantini, D., Marzetti, L. Belardinelli, P., Ciancetta, L., Pizzella, V., Romani, G.L., Corbetta, M., 2010. Tempora dynamics of spontaneous MEG activity in brain networks. Proc. Natl. Acad. Sci. U. S. A. $107,6040-6045$.

de Pasquale, F., Della Penna, S., Snyder, A.Z., Marzetti, L., Pizzella, V., Romani, G.L., Corbetta, M., 2012. A cortical core for dynamic integration of functional networks in the resting human brain. Neuron 74, 753-764.

de Pasquale, F., Della Penna, S., Sporns, O., Romani, G.L., Corbetta, M., 2016. A dynamic core network and global efficiency in the resting human brain. Cerebr. Cortex 26 (10), 4015-4033.

Deco, G., Corbetta, M., 2011. The dynamical balance of the brain at rest. Neuroscientist 17, 107-123.

Deco, G., Jirsa, V.K., 2012. Ongoing cortical activity at rest: criticality, multistability, and ghost attractors. J. Neurosci. 32 (10), 3366-3375.

Deco, G., Jirsa, V.K., McIntosh, A.R., 2011. Emerging concepts for the dynamical organization of resting-state activity in the brain. Nat. Rev. Neurosci. 12, 43-56.

Deco, G., Jirsa, V.K., McIntosh, A.R., Sporns, O., Kötter, R., 2009. Key role of coupling, delay, and noise in resting brain fluctuations. Proc. Natl. Acad. Sci. U. S. A. 106 (25), 10302-10307.
Engel, A.K., Fries, P., 2010. Beta-band oscillations - signalling the status quo? Curr. Opin. Neurobiol. 20 (2), 156-165.

Fischl, B., 2012. FreeSurfer. Neuroimage 62, 774-781.

Fox, M.D., Snyder, A.Z., Vincent, J.L., Corbetta, M., Van Essen, D.C., Raichle, M.E., 2005. The human brain is intrinsically organized into dynamic, anticorrelated functional networks. Proc. Natl. Acad. Sci. U. S. A. 102, 9673-9678.

Fox, M.D., Zhang, D., Snyder, A.Z., Raichle, M.E., 2009. The global signal and observed anticorrelated resting state brain networks. J. Neurophysiol. 101 (6), 3270-3283.

Friston, K.J., 2011. Functional and effective connectivity: a review. Brain Connect. 1 , 13-36.

Friston, K.J., Ashburner, J.T., Kiebel, S.J., Nichols, T.E., Penny, W.D., 2007. Statistical Parametric Mapping: the Analysis of Functional Brain Images. Academic Press.

Friston, K.J., Holmes, A.P., Worsley, K.J., Poline, J.P., Frith, C.D., Frackowiak, R.S.J., 1994. Statistical parametric maps in functional imaging: a general linear approach. Hum. Brain Mapp. 2, 189-210.

Giacino, J.T., Fins, J.J., Laureys, S., Schiff, N.D., 2014. Disorders of consciousness after acquired brain injury: the state of the science. Nat. Rev. Neurol. 10, 99-114.

Gramfort, A., Luessi, M., Larson, E., Engemann, D.A., Strohmeier, D., Brodbeck, C., Parkkonen, L., Hämäläinen, M.S., 2014. MNE software for processing MEG and EEG data. Neuroimage 86, 446-460.

Hall, E.L., Woolrich, M.W., Thomaz, C.E., Morris, P.G., Brookes, M.J., 2013. Using variance information in magnetoencephalography measures of functional connectivity. Neuroimage 67, 203-212.

Handwerker, D.A., Roopchansingh, V., Gonzalez-Castillo, J., Bandettini, P.A., 2012. Periodic changes in fMRI connectivity. Neuroimage 63, 1712-1719.

Hansen, E.C.A., Battaglia, D., Spiegler, A., Deco, G., Jirsa, V.K., 2015. Functional connectivity dynamics: modeling the switching behavior of the resting state. Neuroimage 105, 525-535.

Hindriks, R., Adhikari, M.H., Murayama, Y., Ganzetti, M., Mantini, D., Logothetis, N.K., Deco, G., 2016. Can sliding-window correlations reveal dynamic functional connectivity in resting-state fMRI? Neuroimage 127, 242-256.

Hipp, J.F., Hawellek, D.J., Corbetta, M., Siegel, M., Engel, A.K., 2012. Large-scale cortical correlation structure of spontaneous oscillatory activity. Nat. Neurosci. 15, 884-890.

Hutchison, R.M., Womelsdorf, T., Allen, E.A., Bandettini, P.A., Calhoun, V.D., Corbetta, M., Della Penna, S., Duyn, J.H., Glover, G.H., Gonzalez-Castillo, J., Handwerker, D.A., Keilholz, S., Kiviniemi, V., Leopold, D.A., de Pasquale, F., Sporns, O., Walter, M., Chang, C., 2013. Dynamic functional connectivity: promise, issues, and interpretations. Neuroimage 80, 360-378.

Hutchison, R.M., Womelsdorf, T., Gati, J.S., Everling, S., Menon, R.S., 2012. Resting-state networks show dynamic functional connectivity in awake humans and anesthetized macaques. Hum. Brain Mapp. 34, 2154-2177.

Hyvärinen, A., Oja, E., 2000. Independent component analysis: algorithms and applications. Neural Network. 13, 411-430.

Jensen, O., Bonnefond, M., VanRullen, R., 2012. An oscillatory mechanism for prioritizing salient unattended stimuli. Trends Cognit. Sci. 16 (4), 200-206.

Jung, A., 2017. A fixed-point of view on gradient methods for big data. Front Appl Math Stat 3,18

Kiviniemi, V., Vire, T., Remes, J., Elseoud, A.A., Starck, T., Tervonen, O., Nikkinen, J., 2011. A sliding time-window ICA reveals spatial variability of the default mode network in time. Brain Connect. 1, 339-347.

Klimesch, W., 2012. Alpha-band oscillations, attention, and controlled access to stored information. Trends Cognit. Sci. 16 (12), 606-617.

Klimesch, W., Freunberger, R., Sauseng, P., 2010. Oscillatory mechanisms of process binding in memory. Neurosci. Biobehav. Rev. 34 (7), 1002-1014.

Knyazev, G.G., Savostyanov, A.N., Bocharov, A.V., Slobodskaya, H.R., Bairova, N.B., Tamozhnikov, S.S., Stepanova, V.V., 2017. Effortful control and resting state networks: a longitudinal EEG study. Neuroscience 346, 365-381.

Knyazev, G.G., Savostyanov, A.N., Bocharov, A.V., Tamozhnikov, S.S., Saprigyn, A.E., 2016. Task-positive and task-negative networks and their relation to depression: EEG beamformer analysis. Behav. Brain Res. 306, 160-169.

Kopell, N., Ermentrout, G.B., Whittington, M.A., Traub, R.D., 2000. Gamma rhythms and beta rhythms have different synchronization properties. Proc. Natl. Acad. Sci. U. S. A. 97 (4), 1867-1872.

Krzywinski, M., Altman, N., 2014. Points of significance: nonparametric tests. Nat. Methods 11 (5), 467-468.

Liu, Q., Farahibozorg, S., Porcaro, C., Wenderoth, N., Mantini, D., 2017. Detecting largescale networks in the human brain using high-density electroencephalography. Hum. Brain Mapp. 38, 4631-4643.

Liu, Z., Fukunaga, M., de Zwart, J.A., Duyn, J.H., 2010. Large-scale spontaneous fluctuations and correlations in brain electrical activity observed with magnetoencephalography. Neuroimage 51, 102-111.

Luckhoo, H., Hale, J.R., Stokes, M.G., Nobre, A.C., Morris, P.G., Brookes, M.J., Woolrich, M.W., 2012. Inferring task-related networks using independent component analysis in magnetoencephalography. Neuroimage 62, 530-541.

Marty, B., Bourguignon, M., Jousmäki, V., Wens, V., Op de Beeck, M., Van Bogaert, P., Goldman, S., Hari, R., De Tiège, X., 2015. Cortical kinematic processing of executed and observed goal-directed hand actions. Neuroimage 119, 221-228.

Mary, A., Bourguignon, M., Wens, V., Op de Beeck, M., Leproult, R., De Tiège, X., Peigneux, P., 2015. Aging reduces experience-induced sensorimotor plasticity. A magnetoencephalographic study. Neuroimage 104, 59-68.

Monti, M.M., 2011. Statistical analysis of fMRI time-series: a critical review of the GLM approach. Front. Hum. Neurosci. 5, 28.

Oldfield, R.C., 1971. The assessment and analysis of handedness: the Edinburgh inventory. Neuropsychologia 9, 97-113. 
O'Neill, G.C., Barratt, E.L., Hunt, B.A., Tewarie, P.K., Brookes, M.J., 2015a. Measuring electrophysiological connectivity by power envelope correlation: a technical review on MEG methods. Phys. Med. Biol. 60, R271-R295.

O'Neill, G.C., Bauer, M., Woolrich, M.W., Morris, P.G., Barnes, G.R., Brookes, M.J., 2015b. Dynamic recruitment of resting state sub-networks. Neuroimage 115, 85-95.

O'Neill, G.C., Tewarie, P., Vidaurre, D., Liuzzi, L., Woolrich, M.W., Brookes, M.J., 2017 a. Dynamics of large-scale electrophysiological networks: a technical review. Neuroimage 180 (Part B), 559-576.

O'Neill, G.C., Tewarie, P.K., Colclough, G.L., Gascoyne, L.E., Bae, H., Morris, P.G, Woolrich, M.W., Brookes, M.J., 2017b. Measurement of dynamic task related functional networks using MEG. Neuroimage 146, 667-678.

Pascual-Marqui, R.D., 2002. Standardized low-resolution brain electromagnetic tomography (sLORETA): technical details. Methods Find. Exp. Clin. Pharmacol. 24 (Suppl. D), 5-12.

Pearson, J., Naselaris, T., A.,H.E., Kosslyn, S.M., 2015. Mental imagery: functional mechanisms and clinical applications. Trends Cognit. Sci. 19 (10), 590-602.

Siems, M., Pape, A.A., Hipp, J.F., Siegel, M., 2016. Measuring the cortical correlation structure of spontaneous oscillatory activity with EEG and MEG. Neuroimage 129, 345-355.

Smith, S.M., Fox, P.T., Miller, K.L., Glahn, D.C., Fox, P.M., Mackay, C.E., Filippini, N., Watkins, K.E., Toro, R., Laird, A.R., Beckmann, C.F., 2009. Correspondence of the brain's functional architecture during activation and rest. Proc. Natl. Acad. Sci. U. S A. $106,13040-13045$.

Sockeel, S., Schwartz, D., Pelegrini-Issac, M., Benali, H., 2016. Large-scale functional networks identified from resting-state EEG using spatial ICA. PLoS One 11, e0146845.

Taulu, S., Simola, J., Kajola, M., 2005. Applications of the signal space separation method. IEEE Trans. Signal Process. 53, 3359-3372.

Vander Ghinst, M., Bourguignon, M., Op de Beeck, M., Wens, V., Marty, B., Hassid, S., Choufani, G., Jousmäki, V., Hari, R., Van Bogaert, P., Goldman, S., De Tiège, X., 2016.
Left superior temporal gyrus is coupled to attended speech in a cocktail-party auditory scene. J. Neurosci. 36, 1596-1606.

Vidaurre, D., Hunt, L.T., Quinn, A.J., Hunt, B.A.E., Brookes, M.J., Nobre, A.C., Woolrich, M.W., 2018. Spontaneous cortical activity transiently organises into frequency specific phase-coupling networks. Nat. Commun. 9, 2987.

Vigario, R., Sarela, J., Jousmäki, V., Hämäläinen, M., Oja, E., 2000. Independent component approach to the analysis of EEG and MEG recordings. IEEE Trans. Biomed. Eng. 47, 589-593.

Watson, D., Clark, L.A., Chmielewski, M., Kotov, R., 2013. The value of suppressor effects in explicating the construct validity of symptom measures. Psychol. Assess. 25 (3), 929-941.

Weichwald, S., Meyer, T., Özdenizci, O., Schölkopf, B., Ball, T., Grosse-Wentrup, M., 2015. Causal interpretation rules for encoding and decoding models in neuroimaging. Neuroimage 110, 48-59.

Wens, V., 2015. Investigating complex networks with inverse models: analytical aspects of spatial leakage and connectivity estimation. Phys. Rev. E - Stat. Nonlinear Soft Matter Phys. 91, 012823.

Wens, V., Bourguignon, M., Goldman, S., Marty, B., Op de beeck, M., Clumeck, C., Mary, A., Peigneux, P., Van Bogaert, P., Brookes, M.J., De Tiège, X., 2014a. Inter- and intra-subject variability of neuromagnetic resting state networks. Brain Topogr. 27, 620-634.

Wens, V., Marty, B., Mary, A., Bourguignon, M., Op de Beeck, M., Goldman, S., Van Bogaert, P., Peigneux, P., De Tiège, X., 2015. A geometric correction scheme for spatial leakage effects in MEG/EEG seed-based functional connectivity mapping. Hum. Brain Mapp. 36, 4604-4621.

Wens, V., Mary, A., Bourguignon, M., Goldman, S., Marty, B., Op de beeck, M., Van Bogaert, P., Peigneux, P., De Tiège, X., 2014b. About the electrophysiological basis of resting state networks. Clin. Neurophysiol. 125, 1711-1713.

Zalesky, A., Fornito, A., Cocchi, L., Gollo, L.L., Breakspear, M., 2014. Time-resolved resting-state brain networks. Proc. Natl. Acad. Sci. U. S. A. 111, 10341-10346. 